\title{
La imilla de Paucartambo, Cusco, y la encarnación del entorno: un caso de travestismo y androginia en los Andes ${ }^{1}$
}

\author{
Artículos originales: ANTROPOLOGÍA \\ Recibido: $18 / 04 / 2021$ \\ Aprobado: 12/06/2021 \\ Publicado: 14/10/2021
}

\author{
Yiftah Levin \\ Universidad Hebrea de Jerusalén \\ yiftahl@hotmail.com
}

\begin{abstract}
RESUMEN
El presente artículo postula que la imilla de Paucartambo, Cusco, es un personaje travesti y transgénero que constituye una interpretación del tema de género en los Andes. La pregunta es: ¿quién es la imillla y por qué está tradicionalmente encarnada por un varón? Se argumenta que la participación de la imilla en la actuación del ritual y juego y su simbolismo, es esencial en los Qhapaq Qolla y en la fiesta de la Virgen del Carmen de Paucartambo, como representantes de su entorno cultural. Como personaje liminal, la imilla es única en la fiesta; y constituye un caso único en las representaciones de transgénero y travesti en los Andes, hasta donde conocemos. Finalmente, se argumenta que como personaje transgénero, la imilla encarna la totalidad del concepto de Paucartambo como un núcleo así como esta narrado por los mitos y performado durante la guerrilla.
\end{abstract}

Palabras clave: imilla, Qhapaq Qolla, Cusco, Paucartambo, travesti, transgénero

\section{The imilla of Paucartambo (Cusco) and the milieu incarnation: a case about transvestism and androgyny in the Andes}

\begin{abstract}
The present article proposes that the imilla of Paucartambo, Cusco, is a transvestite and transgender character that constitute an interpretation of the theme of gender in the Andes. The question is: who is the imilla, and why is it traditionally embodied by a man? It is argued that the participation of the imilla in the performance of ritual and play and its symbolism, is essential in the Qhapaq Qolla and the fiesta of the Virgen del Carmen of Paucartambo, as representative of its cultural environment. As a liminal character, the imilla is unique in the fiesta; and it constitute a unique case in the transvestite and transgender representationes of the Andes, as far as we know. Finally, it is argued that as a transgender character, the imilla embodies the entire concept of Paucartambo as a nucleus, as it is narrated in the myths and performed during the guerrilla.
\end{abstract}

KeYwords: imilla, Qhapaq Qolla, Cusco, Paucartambo, transvestite, transgender

1 Agradecimientos: Quisiera expresar mis agradecimientos a los miembros anteriores y presentes de los Qhapaq Qolla, al antropólogo Santiago Palomino Coll. Cárdenas y especialmente al artista mascarero Mario Palomino Coll. Cárdenas. 


\section{Qhapaq Qolla-participación ritual y estructura}

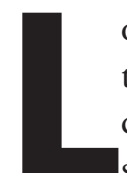

os Qhapaq Qolla de Paucartambo constituyen una de las tropas de danza ritual más destacadas en el Cusco, con un estatus que se ha intensificado luego de que la fiesta de la Virgen del Carmen se haya popularizado. Además de la fiesta del 16 de julio, participan también en el peregrinaje al santuario del Señor de Qoyllur Rit'i, en la provincia contigua de Quispicanchi. Aunque en ambos lugares de peregrinación participan numerosas tropas, en estos hay protagonismo de la nación Paucartambo y de los Qhapaq Qolla en particular. Así, analizar la performance de la tropa permite un conocimiento de ambos peregrinajes y su contexto.

Juntos con los Qhapaq Ch'uncho y los Qhapaq Negro, los Qhapaq Qolla constituye la danza más importante en términos rituales en Paucartambo, con un estatus que está narrado por las historias o mitos que cuentan la llegada de la imagen de la Virgen al pueblo en el siglo XVII (Villasante Ortiz, 1989; Cánepa, 1998), igual como en el proceso ritual de la fiesta. El traslado de la imagen del Qollao o de Q'osñipata a Paucartambo involucra una rivalidad de los habitantes Qolla o Ch'uncho hacia el pueblo, una rivalidad que luego se transformó en asistencia, devoción y posición privilegiada en la fiesta. Esa rivalidad está incorporada en la guerrilla, del 17 de julio, pero es una rivalidad transformada. La guerrilla es una actuación que representa una lucha entre los Qollas y los Ch'unchos, en donde los Ch'unchos siempre matan a los Qollas y a su alcalde, y donde el rey Ch'uncho se lleva la imilla, un personaje femenino. ¿Quién es la imilla, por qué la raptan, y por qué está tradicionalmente encarnada por un varón? Basado en un trabajo de campo realizado en el año 2017, argumento que la participación de la imilla en la actuación única del ritual y juego de la tropa y su simbolismo es esencial en los Qhapaq Qolla y en la fiesta de la Virgen del Carmen de Paucartambo.

Las oportunidades limitadas de empleo en Paucartambo causaron procesos de emigración, una situación que se incrementó después de la Reforma Agraria (Van Den Berghe \& Primov, 1977). En 1972 el alcalde de Paucartambo declaro a la Virgen del Carmen patrona del pueblo, después de que las tropas de danza ganaran el trofeo Inti Raymi en el Cusco, y tras ganar la competencia por seis años seguidos
(Villasante Ortiz, 1989). La mayoría de los integrantes de las tropas eran paucartambinos, entonces residentes en el Cusco (Testimonio de $\mathrm{Mario}^{2}$ ), marcando así la fiesta como un peregrinaje de los emigrantes paucartambinos. La palabra Qolla indica una conexión con la región Qollasuyo, la que extiende del Cusco hacia el sudeste, mientras Paucartambo está en la región Antisuyo, creando así la división de hanan y hurin $^{3}$ (Zuidema, 1964); la región Qollao está identificada con la zona sur, a través de abra de La Raya, como una división geográfica y cultural entre Cusco y Puno (Poole \& Zuidema, 1982). Más aun, el término Qolla adapta un significado simbólico de los habitantes de la puna que vienen como foráneos por motivos de comercio a varios santuarios en Cusco (Gow \& Condori, 1982; Poole, 1982; Morote Best, 1988: 1-38). Así, en las historias de Paucartambo, la conexión con Puno está relacionada con el movimiento de comercio y peregrinación de los Qollas. Como las demás tropas, los Qollas son paucartambinos nativos o relacionados por parentesco con paucartambinos, aunque menos de la mitad residen actualmente en Paucartambo. Este dato enfatiza el carácter foráneo de los Qollas, que se está reafirmando por procesos de migración. Así, los Qollas no solamente representan un foráneo simbólico, sino que se están convirtiendo en peregrinos que vuelven a la tierra natal. Usando los términos de Coleman y Eade (2004), los Qollas convierten un movimiento metafórico en movimientos de migración y peregrinaje a Paucartambo, mientras mantienen Qoyllur Rit'i como peregrinaje. La participación de los Qollas en ambos peregrinajes, Paucartambo y Qoyllur Rit'i, refleja una percepción de la vida religiosa y cultural, como una dualidad que mientras complementa símbolos cristianos con andinos, organiza el mundo social y la identidad de los paucartambinos al realizar su ritual en tierra ajena, provincia de Quispicanchi. Esta dualidad está reflejada por ellos mientras actúan una identidad liminal dentro de una sociedad en proceso de transición.

2 Los testimoniantes que cito son: Amador (en sus setenta, alcalde de Qolla 1972-92); Braulio (en sus sesenta, alcalde de Qolla 19931995); David (en sus veinte, imilla en 2016-2018); Edy (en sus treinta, danzante de Qolla); Francisco (en sus setenta, imilla 1952-1956); Frid (en sus cincuenta, alcalde de Qolla 1996-1998); Margot (en sus cuarenta, imilla 1996); Mario Palomino (en sus sesenta, danzaba Saqra, Qhapaq Negro, Qhapaq Qolla, Contradanza); Martín (en sus treinta, Qolla, imilla 2009-2015).

3 Hanan-arriba (Antisuyo y Chinchaysuyo); hurin-abajo (Qollasuyo y Contisuyo). 
Los Qollas se describen como «seres antropomorfos, mitad hombre, mitad llama» (Testimonio de Edy). Esta concepción simbólica está reflejada en su coreografía y en su organización social. El caporal se titula alcalde y se compara con el hayñachu, el jefe del rebaño de llamas. El alcalde es la mayor función de la tropa y su autoridad es indiscutida. Desde 1990 es elegido por la tropa para un periodo de tres años, los que se pueden prolongar solo una vez. El alcalde escoge su imilla entre los miembros de la tropa o trae una de afuera, y ella lo sigue en la jerarquía. Otros personajes son el quita Qolla y los llameros. El quita es comparado al quita llama ${ }^{4}$ y se considera como un Qolla incivilizado, que va fuera de la cuadrilla, y que puede hacer sedición al hayñachu. Los llameros, acompañados con el hayñachu del rebaño, funcionan como grupo satélite de la tropa que camina por el pueblo en los demás kargohuasi 5 . La ropa del Qolla expresa un simbolismo de camélido: las quellas, camélidos disecados, expresan su poder económico; el q'epe de vicuńa cuelga de su espalda, y pukuchos de alpaca en su pecho. Su máscara, el waqollo, además de cubrir la cara y así su identidad, simula la cara de un camélido. Finalmente, los Qollas adaptan también el personaje de la llama: «no está quieto; solo un rato puede estar tranquilo y después (no esta) ...” (Testimonio de David).

Según Lévi-Strauss (1966: 191-216), las comunidades metafóricas de animales son comunidades que, por ser independientes de nuestra comunidad, están comprendidas como otra sociedad, que es similar a la nuestra en términos de valores compartidos. En los Andes los camélidos viven una existencia paralela al hombre y se encuentran en ciertas ocasiones constituyendo una sociedad metafórica (Sallnow, 1987: 131-136). La relación metafórica entre ambas sociedades está presente en la cultura popular (Figueroa, Nishiyama y Villanueva, 1961) y folklórica (Arguedas, 1964; Itier, 2004: 185-187), lo que connota una existencia liminal, que se manifiesta en momentos post mortem o después de infringir la separación entre las sociedades. Los Qhapaq Qolla se conceptualizan como medio camélidos, así que re-

4 En el rebaño de llamas «el quita no quiere hacer caso a la comparsa. El quita puede hacer golpe al hayňachu porque no va ser el líder. Al quita no se le puede dominar, se va de la fila. No quiere pertenecer al grupo. Esta ausente» (Testimonio de Edy).

5 Casas de cargo. Lugar donde se reúnen las tropas de danza. presentan un ser liminal. Para los paucartambinos el Qolla en su esencia es «jocoso, jodido, un plaga», que adapta una labor responsable en la fiesta y en la sociedad, como un «equilibrio también de niño y serio", o una forma de juego. Así, ser Qolla significa estar entre la sociedad y no estar en la sociedad; es estar en una situación liminal constante, en una identidad liminal de danzante en su sociedad, que está actuada y encarnada en este personaje. Según Turner (1982), el juego es una parte esencial del personaje del danzante que se personifica durante el proceso ritual. En Paucartambo, la función liminal del personaje imilla, sobre todo en la performance del juego, se marca o evidencia palmariamente, dada la condición liminal del proceso ritual que significa la fiesta y todo lo que representa ésta.

Además de ambos peregrinajes, los Qollas cumplen su responsabilidad con el pueblo en reuniones a lo largo del año, lo que hace constituir a la tropa como una familia. En esta familia el alcalde es el padre y la imilla la madre. La pareja imaginaria está presente en la actuación del Qolla y en la imagen corporal de la imilla.

\section{Performances de ritual y juego}

La cosmología andina se basa, desde la época prehispánica, en un equilibrio que mantiene fuerzas duales que se complementan y se contrarrestan (Classen, 1990; Isbell, 1976); y así encarnan potencial de fertilidad y capacidad de reproducción como una forma de organizar el mundo social y como medio de complementariedad con el mundo natural (Isbell, 1997). El cuerpo humano sirve como modelo para el cosmos y como mediador entre las fuerzas, por su estructura y funciones (Classen, 1990). Estas oposiciones están comprendidas como masculino, con derecha, lo alto y exterioridad, mientras femenino se vincula a izquierda, abajo y el interior. El primer grupo está asociado con estructura, claridad y fuerza fertilizante; el segundo, con fluidez, oscuridad y fecundidad. Normalmente el primer grupo se comprende como superior; pero es complementario con el segundo; y cada uno es esencial para la existencia del otro (Ackerman, 1991; Classen, 1990). Yanantin expresa pares o unión fundamental e integridad, mientras karihuarmi expresa la unión hombre-mujer, 
y Wiracocha representa el concepto de karihuarmi que encarna esa idea original de integración (Classen, 1990). Esa unión se ha expresado en esculturas andróginas que eran veneradas y luego destruidas durante la conquista (de Arriaga, 1968: 79 citado por Classen, 1990: 42).

La imilla y el alcalde cumplen el karihuarmi en su existencia dual y la imilla lo cumple en su cuerpo: personaje femenino que está encarnado por un danzante masculino, vestido con prendas femeninas, mientras una tela negra cubre su rostro, símbolo femenino, en comparación al waqollo blanco del Qolla. Hombre que es una mujer y una mujer que es un hombre, una identidad doble que no tiene identidad, no tiene rostro. La tropa está organizada jerárquicamente en dos líneas, así que el primer sustituto es el capitán de la fila derecha; y le sigue el capitán de la fila izquierda. La estructura de dos líneas es mantenida durante toda la fiesta ${ }^{6}$.

Propongo el análisis de la presentación de los Qollas por su composición, de tres categorías de performances: de ritual y de juego y de ritos de paso, que se distinguen dado el diverso papel de la imilla dentro de la tropa. Si bien debe entenderse que los límites de lo ritual, lo religioso y lo lúdico no tienen límites perfectos. Las categorías tipológicas de ritual y juego son válidas en el papel, pero la realidad siempre tiene continuidades, y el juego o lo lúdico tiene de ritual; el ritual tiene de lúdico; el teatro tiene de sagrado; etc. Los rituales son performances que tratan de la Virgen y las relaciones con ella en una forma directa; mientras que el origen católico de los juegos es ambiguo. Además de ser ritos de iniciación y de transición (Van Gennep, 2004), los ritos de paso tienen en este contexto un papel mediador, y tienen caracterizaciones de ambas performances, de ritual y de juego. Argumento que la estructura temporal y espacial de la fiesta está relacionada con la performance del Qolla del ritual y juego y con el papel de la imilla en cada categoría de performance.

Las performances de ritual son tres. La entrada está cantada y danzada durante el primer día de la fiesta, 15 de julio al mediodía en el templo y en medianoche en la serenata, frente al templo. El para el

6 En las reuniones del kargohuasi están sentados en dos líneas y la comida se sirve primero al alcalde y la imilla y luego a la fila derecha y la fila izquierda; cuando se acercan a las tumbas en la romería; en su coreografía; y en el yawar unu trabajan en pares opuestos definidos. día, es cantado en la mañana del 16 de julio, durante la misa y en la procesión. La bendición es cantada el 17 de julio en la romería, en la segunda procesión, y el 18 de julio, en la bendición. En las tres coreografías la tropa danza en dos líneas, mientras el alcalde y la imilla se están moviendo en direcciones opuestas entre las líneas, observándolas. Los movimientos lentos y sincronizados, el canto en quechua y la música, hacen que estas performances se sientan sagradas. Como movimientos performativos (Coleman \& Eade, 2004) están presentadas dentro y fuera del templo y en las procesiones; así, marcan las rutas sagradas del pueblo y constituyen un espacio social. Como movimientos incorporados (Coleman \& Eade, 2004), estimulan la fe y devoción que los Qollas expresan hacia la Virgen, y el sacrificio involucrado. En el paso de lo impuro a lo religioso se involucran transformaciones en el manejo y trato del cuerpo en el peregrinaje al Qoyllur Rit'i, los bautizos y el yawar unu. Además del sacrificio corporal, hay un sacrificio religioso, expresado en la bendición, con la lamentación por la separación de la Virgen, de los demás Qollas, y del pueblo, enfatizándose así el carácter peregrino del Qolla. Mientras cantan esta parte, ambas líneas se acercan y se abrazan, presentando así la función complementaria del yanantin.

Como performances de rito de paso identifico al bautizo, romería y yawar unu, que están presentados entre performances de ritual y juego o en un espacio liminal. El bautizo y la romería están performados en el cementerio. Los Qollas son bautizados por el alcal$d e$ cuatro veces en su primer año, dos en Qoyllur Rit'i, y dos en Paucartambo. En Paucartambo son bautizados el 16 de julio, después de la misa, y el 17 de julio, después de la romería. Aunque el nombre indica una conexión fuerte con el cristianismo, no involucra una oración expresiva, empero su realización con intención religiosa por el alcalde se cree recompensada. En Qamawara, Cusco, en carnavales, a los camélidos se les practica el tink'ay, que consiste en marcárseles la espalda, con el objetivo simbólico de propiciar la fertilidad de los animales adultos, y bautizar a los animales jóvenes (Sallnow, 1987: 132-136). Se trata de un ritual que lee a los animales como humanos. En el caso de los Qollas, la lectura es inversa: a los hombres se les lee como animales: sus espaldas son marcadas con azotes. Los Qollas adultos se azotan como forma de castigo, sin echárseles cerveza encima. En la ro- 
meria, la integración entre huaynos de amor, chistes y risa, se mezcla con el canto de la bendición a los compañeros difuntos. Los principios contradictorios que aparecen en las parodias en los Andes, como en la romería y el bautizo, no tratan de desarrollar una síntesis, sino que están guardados como intactos y siguen coexistiendo dentro de un conflicto insoluble (Ackerman. n.d: 12), intensificando así la transición entre ritual y juego, como parte de la presentación del Qolla.

El yawar unu es un ritual de transición que se desarrolla dos veces —el 15 de julio, después de la entrada; y el 18 de julio, después de la bendición-. Le siguen las performances de juego, y así se define como un ritual mediador. El ritual empieza cuando el alcalde y la imilla entran entre las líneas, se abrazan frente al templo o la demanda de la Virgen, hacen una reverencia, retroceden y repiten esto tres veces. El alcalde se mantiene con los brazos cruzados y la imiIla le azota; cambian de posiciones y el acto se repite tres veces. La música se acelera, los Qollas se mueven inquietos y se azotan entre sí hasta que un Qolla los separa. Se abrazan y hacen una reverencia hacia el templo. Luego, los demás Qollas presentan el ritual y entonces la imilla los separa. Según los Qollas, el yawar unu sirve como ocasión de cerrar cuentas personales, un momento de guerra donde algunos merecen ser azotados: «Hay gente como una familia que a veces curan sus rencillas así de esa manera, ¿no? Yo te quiero sacar porque te quiero sacar, hacemos; después vemos qué pasa.» (Testimonio de David). En esta familia la imilla es comprendida como la madre que separa a los hermanos en disputa. El ritual es el tiempo apropiado para liberar conflictos interpersonales: empieza y acaba con un abrazo frente a la Virgen como testigo. Así, funciona como un ritual de sacrificio (Girard, 2005) que protege a los Qollas de su propia violencia para restaurar armonía y reforzar la estructura social. El yawar unu puede ser entendido como un tinkuy, a modo de un evento organizado que toma interés personal y colectivo, que se realiza para resolver disputas (Sallnow, 1987). También puede verse como una preparación para la guerrilla, demostrando fuerza y sacrificio, como los Qollas cuentan que históricamente lo hacían para prepararse para la lucha.

Las performances de juego están compuestas por la coreografía de juego y las performances de bosque, q'onoy y la guerrilla?. La coreografía tiene lugar en la plaza el 15 de julio; y el 18 de julio, al frente del templo después del yawar unu. Está compuesta por una secuencia de siete escenas, que tienen un carácter cómico: los movimientos son más rápidos, la imilla tiene un papel específico y separado del alcalde, la Virgen no está mencionada, ni su veneración. En el chinka-chinka los Qollas hacen un círculo mientras uno le persiga a la imilla, que escapa entrando y saliendo del círculo. Cuando ésta es capturada bailan un huayno en el medio mientras la tropa corre de un lado a otro cantando. Luego, el Qolla abraza la imilla. La canción expresa un noviazgo donde un mozo se está perdiendo por la culpa de una mestiza joven. En el iñiñi los Qollas rodean a la imilla, que está sentada haciendo la señal de la cruz con sus dedos, ésta se voltea salta y los Qollas voltean y escupen hacia el público. Este acto es descrito como una imitación directa de las llamas. En la pukacinta, cinta roja, las dos líneas bailan agarradas de las manos acercándose y alejándose de la línea opuesta en una forma desigual imitando el movimiento de la cinta en el viento. Las letras describen la conjugación imposible de una cinta roja con la verde. Las cintas y las líneas pueden ser comprendidas como representación de fuerzas duales que se complementan y se contrarrestan en un intento de construir una unidad, como en la bendición. Agarrándose de la mano los Qollas bailan en una fuga de huayno, circulando por la plaza. Las letras expresan el placer del joven cargando a su mujer por las rocas. Ahí, la tropa se cae al suelo y la imilla pasa uno por uno levantando su pollera, mientras los Qollas le repelen por el olor que se les dispersa y se levantan. En el chaskaschay, mi estrella, los Qollas bailan saludando con sus pukuchos, mientras la imilla hace lo mismo al medio. Mientras cantan se acercan haciendo gestos, en grupo o individualmente, levantando los pukuchos. Las letras cuentan como el Qolla le invita a la joven a su tierra y reprocha que aunque duermen juntos no se embaraza. Luego en el charqui-tauqa, pila de chalona, los Qollas se amontonan horizontal y verticalmente, mientras el alcalde y el primer capitán los persiguen. Entonces, esos dos saltan y finalmente la imilla se para encima hilando. La imilla y el alcalde arrastran al suelo a los Qollas, y ahí la imilla pasa

El bosque y el q’onoy no serán analizados porque no los considero significativos al tema de la imilla. 
de nuevo haciéndoles levantar con su olor. Este acto representa la carne desecada que los Qollas llevaban a Paucartambo. Así, en unos actos imitan la actitud de la llama y en el último acto le imitan su transformación en carne.

Un aspecto evidente que demuestra la diferencia entre las performances de ritual y juego es el papel de la imilla. Mientras que en el ritual actúa como un reflejo del alcalde, en el juego se transforma en una joven traviesa y deseada. Así, la imilla representa un personaje complejo que refleja en muchos aspectos el carácter liminal de los Qollas. En la diferencia entre ambas performances está puesto el núcleo de la presentación notable del Qolla en Paucartambo. Los Qollas describen la integración de ritual y juego como «el equilibrio también de niño y serio... es que la llama no está quieta, solo un rato» (Testimonio de David). Entonces, ser serio y alegre, o ser niño y adulto, es como ser una llama, un personaje liminal. El uso de la máscara bestial connota a la gente la característica esencial de la bestialidad de la máscara y la identidad anónima de la persona enmascarada (Ackerman, n.d). Luego, estando enmascarados actúan como espejos anónimos; así, los Qollas reflejan al espectador paucartambino. "Los dos son espacios muy diferentes que hacen al Qolla... nosotros como Qollas somos más la representación del pueblo. En las actividades que hacen... una fiesta es alegría; muere alguien, es tristeza... Parte de eso está dentro lo que es la coreografía del Qolla... de repente es algo más completo que se ha hecho con el Qolla» (Testimonio de Frid). Handelman (2004) sugiere que el ritual es la representación de la vida que produce sus propios símbolos, reflexiones que representan temas de la vida y así la controlan. Así, el Qolla es la representación fiel de su entorno, reflejándolo y modelándolo.

Los Qollas tratan su presentación como puesta en escena. En este sentido todo el pueblo puede ser comprendido como un escenario, donde los actores crean un espacio social propio por su participación en el lugar (Hastrup, 1998). Los Qollas encarnan una contradicción de aspectos y papeles dentro de la fiesta, que en su esencia es una representación del pueblo. Siendo agentes dobles reflejando hacia ellos mismos y sus personajes (Hastrup, 1998), ellos presentan un personaje juguetón. Las diferencias entre juego y ritual contribuyen al orden social con la fusión de moralidad e inmoralidad (Handelman, 1977). Así, la integración de los participantes como una comunidad está ejecutada por la conjugación de devoción y entretenimiento. Además, si un significado social está encarnado en dinámicas corporales (Sklar, 2001: 176-195), entonces las diferencias entre ritual y juego marcan espacio y tiempo. Las performances de ritual están ejecutadas dentro o fuera del templo; las del juego están ejecutadas en el mismo lugar después de las performances mediadoras o en un espacio diferente, la plaza. Las performances mediadoras están actuadas también en el cementerio que media entre la vida y la muerte, y entre el pueblo y el mundo exterior.

La segunda secuencia de juego es el q'onoy, bosque y la guerrilla, que son ejecutadas en la plaza. La guerrilla es una secuencia dramática que sigue la segunda procesión en el 17 de julio. La ejecución está finalmente reducida a una batalla entre los Ch'unchus y los Qollas, que siempre acaba con la muerte de los Qollas y con el rapto de la imilla por el rey Ch'unchu.

El concepto de batallas rituales en los Andes contemporáneos ha sido analizado extensamente (Hopkins, 1982; Isbell, 1997; Platt, 1987, Sallnow, 1987; Urton, 1993; Zuidema, 1991). La guerrilla está influenciada por tres instituciones culturales: batallas teatrales, tinkuy y el mito de Inkarri y Qollari. Las batallas teatrales se dividen en dos: las batallas de moros y cristianos y la trágica muerte de Atahualpa ${ }^{8}$. Por otro lado el tinkuy, encuentro, está relacionado con la división de hanan-hurin, como un ritual de reintegración de fuerzas opuestas que tiene su origen en los andes precolombinos (Classen, 1990; Hopkins, 1982; Platt, 1987; Sallnow, 1987; Urton, 1993; Zuidema, 1991). Es un encuentro entre personas similares que involucra un acto violento (Sallnow, 1987: 136-140). La fertilidad de la tierra está relacionada a la fertilidad animal (Sallnow, 1987) y la fertilidad humana (Hopkins, 1982; Platt, 1987); así, mayormente los contendientes son jóvenes; la sangre derramada de las víctimas está enfatizada, y las solteras del lado derrotado son raptadas (Hopkins,

8 Las batallas de moros y cristianos es una dramatización de la totalidad del mundo espańol y las relaciones que representa (Bataillon, 1995) como una batalla entre católicos, y pecadores, los cuales estos encarnan en moros o indígenas (Bataillon, 1995; Zuidema, 1991). La trágica muerte de Atahualpa narra mediante una perspectiva indígena el encuentro violento de los incas con los espańoles como una tragedia que afectó profundamente el orden cósmico, y cuya armonía será restablecida por el retorno del Inca (Wachtel, 1973). 
1982; Sallnow, 1987). Las oposiciones duales del tinkuy corresponden a un sistema recíproco de intercambio entre las comunidades en las diferentes alturas (Murra, 1997). Esta oposición es expresada en los mitos de Inkarri-Qollari. En las versiones recogidas en el Cusco, Qollari se incorpora al Inkarri como una oposición complementaria que representa la división étnica y ecológica entre Cusco y Puno, marcada por el abra La Raya (Hopkins, 1982; Muller \& Muller, 1984; Pease, 1973). Ambos reyes realizan varias competiciones, en las cuales Inkarri siempre derrota a Qollari. Así, aunque están ejecutadas teoréticamente entre dos fuerzas iguales, en realidad está reflejada una asimetría constante.

Así, La guerrilla es una presentación teatral que se reduce a una batalla que representa la división entre Puno y Q'osñipata, o las regiones históricas de Qollasuyo y Antisuyo respectivamente. Los informantes argumentan que la batalla representa un evento histórico que ocurrió entre Qollas y Ch'unchus en el Corpus Christi. Ambos vinieron a Paucartambo, así que están conceptualizados como foráneos, aunque los Ch'unchus se caracterizan más por la región paucartambina. La identificación de Paucartambo con los Ch'unchus pudiera ser entendida desde el mito de Inkarri o desde la batalla de los españoles contra los incas en Vilcabamba, parte del Antisuyo, donde por cierto el movimiento Taki Ongoy fue derrotado (Duviols, 1977; Millones, 1973). Además, durante la conquista española las fuerzas del Inca Manco se refugiaron en la cordillera oriental de Paucartambo y Quispicanchi (Villasante Ortiz, 1989). En este caso, ni Qolla ni Ch'unchu pueden ser referidos como pecadores, sino como dos mitades que luchan por la posesión de la Virgen. En un tinkuy en Potosí dos mitades opuestas luchan en el Corpus Christi como «soldados del Cristo» por una «justicia balanceada» (Platt, 1987). Así, se puede argumentar que Ch'unchus y Qollas se presentan como «soldados de la Virgen».

La secuencia de la fiesta supone representaciones del pasado, con aquello que representa la guerrilla, y representaciones del presente, con el ritual, la veneración a la Virgen. La presentación simultánea asegura la presencia de la Virgen en Paucartambo; y la realización de las dos performances se conjugan en un equilibrio que crea la historia, el presente y el futuro del pueblo, como un ouroboros que se refuerza por la participación. Así, las performances de ritual podrían ser vistas como representación teatral. La separación temporal y espacial entre ambas performances impide la confusión. Y la guerrilla mantiene los papeles complementarios que aseguran que «suceda cada año igual... estamos cerca a la Virgen, pero más cerca están los Ch'unchos, porque ellos son los guardianes. Como han ganado ellos, siempre van a estar a lado de la Virgen... y nosotros como perdedores tenemos que ir un poquito alejados... pero sí tenemos esa facultad de llegarle a la Virgen con nuestros cánticos... nuestro baile que hacemos» (Testimonio de Frid). De esto, debe entenderse que las performances corporales no están hechas con un fin visual, sino como medio de incorporar fe. Entonces, el juego no funciona solamente como opuesto al ritual (Handelman, 1977), sino por su conjugación se incorporan al núcleo de la sociedad y religión paucartambinas, definiendo jerarquías, estatus y papeles dentro de su relación y proximidad con la Virgen del Carmen, la representación más alta de la identidad paucartambina.

Pero, un problema aparece cuando la imilla es raptada por el rey Ch'unchu: ¿Por qué la imilla es raptada en una guerrilla que representa la lucha mítica por la Virgen del Carmen? Mas aun siendo que el supuesto personaje femenino está encarnado por un varón.

\section{La imilla}

Imilla es una palabra aymara que refiere a una joven casadera, y una variedad de papa que se cosecha temprano (Cánepa, 1998: 99) de color oscuro. Productos agrícolas como papa, maíz, haba y qañiwa están relacionados con diferencias étnicas entre valle y puna (Hopkins, 1982; Pease, 1973; Urton, 1993). Según Isbell (1997) los tropos femeninos de sexualidad y reproducción definen la existencia durante la vida y así intensifican el orden social. La vida empieza en un estado húmedo. La diferenciación entre los sexos aumenta hasta la adolescencia. En la madurez las fuerzas de reproducción están destinadas hacia un fin reproductivo; la sociedad se enfoca en la fase reproductiva de la madre, warmi. En la vejez ambos sexos se convierten en chuño y pierden su género específico, como la papa desecada pierde su humedad (Isbell, 1997). Según estos tropos, la imilla es una 
papa tempranamente cosechada que está puesta en la fase reproductiva, como una warmi. La importancia de este paralelismo es evidente en la coreografía de juego cuando la imilla toma el papel de una joven?.

El rapto de la imilla como símbolo femenino está explicado por el tinkuy que relaciona fertilidad humana con la fertilidad de la tierra (Hopkins, 1982). En diferentes lugares en el Cusco las batallas rituales acabaron con el rapto de mujeres por los varones del lado triunfador (Hopkins, 1982; Sallnow, 1987; Urton, 1993). En los mitos de Inkarri-Qollari, Inkarri sedujo a la mujer o hija de Qollari, la violó y la hizo sangrar, mientras que Qollarri no pudo seducir a la mujer de Inkarri (Pease, 1973: 87-101). El mito enfatiza la asimilación de fertilidad de la tierra con la fertilidad humana, por el intercambio de comida y mujeres, y el derramamiento de sangre de vírgenes. Adicionalmente, el mito connota la guerrilla por el rapto de una mujer Qolla.

El personaje de la imilla tiene diversas interpretaciones, que varían desde las percepciones de cada imilla y alcalde. Parece que cada uno aumenta otra dimensión e interpretación, que cambia de acuerdo con la época. La imilla es vista como «la pareja del caporal... esos ordenan todo" (Testimonio de Francisco), o un «toque femenino que tiene que haber... Necesariamente debió de haber una mujer y la imilla es esa representación» (Testimonio de Frid), y dentro de la estructura familiar como «una madre se porta ante sus hijos» (Testimonio de Amador). Pero esta mujer es particular, «es una señora que tiene un corazón lindo, la mamacha... porque la imilla representa pues todo, todita la historia de Paucartambo... esta señora, señorita, es la muy lo único correteador, una persona muy vulgar» (Testimonio de Braulio). Algunos la aprehenden como la pastora de llamas, pero también como "un espíritu entre las llamas. Porque si los Qollas son las llamas que están detrás de un hayñachu... no creo que necesitan una pastora, ¿no? A una pastora no creo que le hagas un yawar unu... yo creo que es el espíritu femenino de todos los Qollas... como una representación de una mujer en sí. Porque un momento representa a la madre, en un momento representa a la Virgen, en la guerrilla por ejemplo... el Qolla es tan masculino, tan chus-

9 El vínculo de la imilla al Qollao aparece también en Potosí, donde en el ritual del hayñachu, dos llamas son agarradas por jóvenes llamadas imilla wawas (Platt, 1987). co, que también tendrá su lado femenino, como el yanantin» (Testimonio de David). El yanantin es el término para parejas, que se encarna en el karihuarmi. Aquí la imilla se entiende como complemento de toda la tropa.

Algunos aprehenden la imilla como una representación de la Virgen del Carmen que participa en la guerrilla en la disputa entre los Qollas y los Ch'unchus. Eso puede ser explicado por la creencia de que la Virgen puede encarnarse por la personificación de distintas mujeres (Morote Best, 1988: 111-151), como las diferentes representaciones de la imilla. Una danzante de imilla va más allá y reclama que «la imilla es fiel representante de la Virgen del Carmen... Nosotros nos encarnamos en la Virgen del Carmen» (Testimonio de Margot). Así, la singularidad de los Qollas de Paucartambo se enfatiza, mientras que «las demás naciones... ellos lo bailan por bailar porque ellos no representan nada. Es la única Virgen del Carmen en Paucartambo que había habido una guerra (donde la Virgen participó), una lucha entre los qollasuyos y los antisuyos» (Testimonio de Margot). Aquí la singularidad de Paucartambo está enfatizada contra los demás pueblos, al encarnar el conflicto mítico e histórico de AntisuyolQollasuyo, Inkarril Qollarri, CuscolPuno, reflejando su papel mediador a través de la representación de la Virgen del Carmen. El programa colonial de asentamiento fue interpretado por las ideas de espacio sagrado y tiempo ritual de los Andes, que expresan una perspectiva étnica de la reproducción social y ecológica (Platt, 1987), que está representada por medio del encuentro de Ch'unchus con Qollas en Paucartambo. Los Qollas hacen lo mismo con el personaje vago de la imilla y su representación de la Virgen. Así, como significante flotante (Lévi-Strauss, 1987: 63-64), la imilla permite varias interpretaciones por sus representantes a pesar de las posibles contradicciones. Es un personaje cuya encarnación es una parte esencial de su existencia, como Hastrup (1998) argumenta, que el mundo de los actores es caracterizado por una reflexión explícita, mientras que tienen que involucrarse en este proceso hacia el personaje.

La cara cubierta hace a la representación de la imilla aun más vaga: «representa a todas las mujeres del Qollao, puesto que no tiene rostro definido» (Testimonio de Martín). Otra explicación: «Llevamos un tul negro... para que no nos reconozcan nuestro 


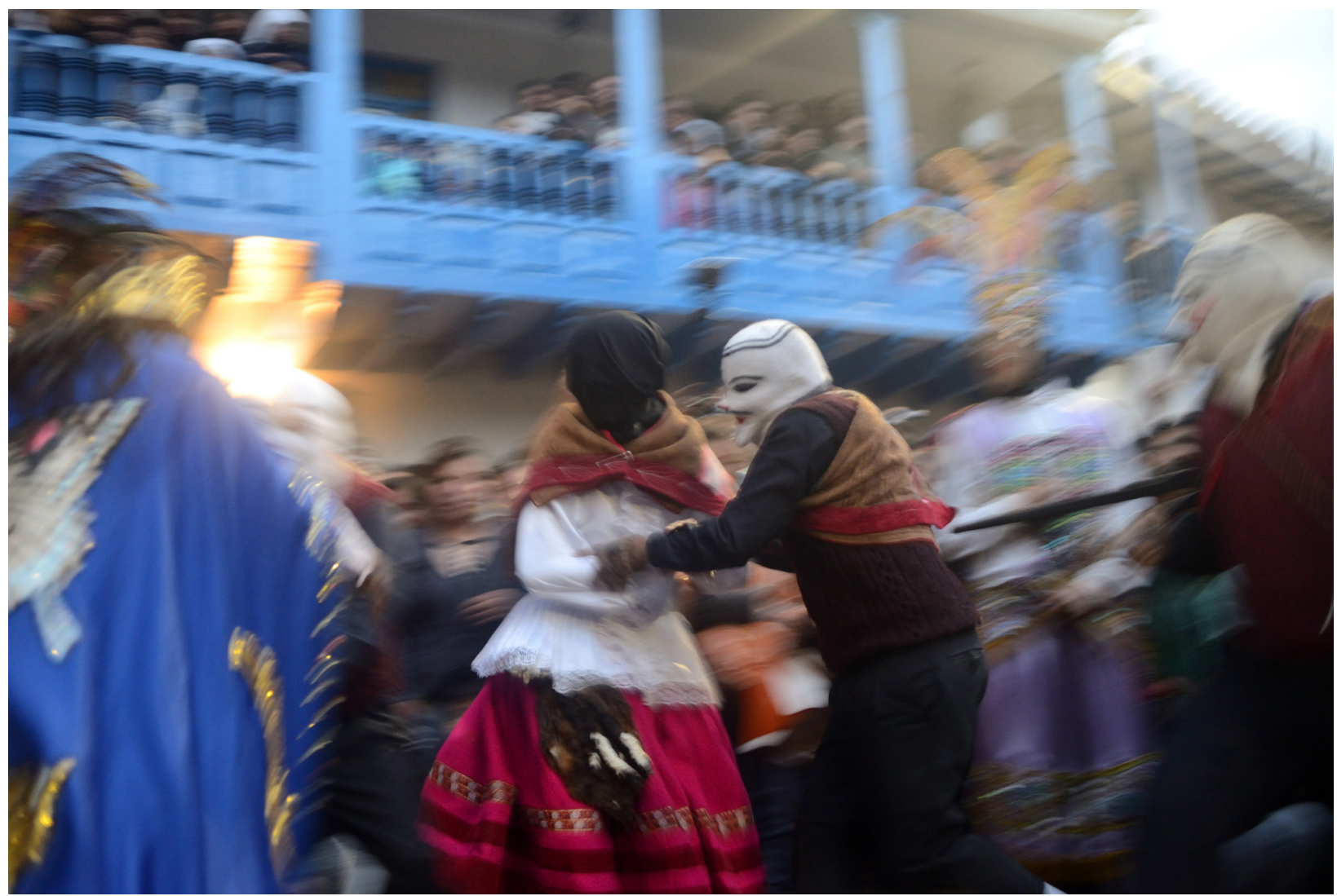

La imilla (al centro) acompañada por un Qolla, cuando es raptada por el rey Ch'unchu (a la izquierda) durante la guerilla.

Paucartambo, 17 de julio, 2017. Fotografía de Yiftah Levin.

rostro, para que no sepan si es varón o es mujer... cuando entraban al templo, las señoras antiguas entraban con velo, ¿no cierto? Es una representación de la religión católica» (Testimonio de Margot). Entonces, la tela negra tiene un atributo ambiguo ${ }^{10}$. Además, oposiciones de género están marcadas en los Andes por un simbolismo de colores: claro para hombre y oscuro para mujer, siendo así opuesto al waqollo blanco de los Qollas. En Lima del siglo XIX, las mujeres se vestían con saya y manto, lo que les dejaba solamente un ojo descubierto para poder caminar. Lo usaban para circunvalar las formalidades y poder moverse y hablar sin ser reconocidas, mirando fijamente a los hombres mientras permanecían incógnitas (Classen \& Howes, 2014). Esta habilidad de presentarse como un personaje ambiguo permite a los danzantes actuar mientras no actúan y adaptarse a un personaje de karihuarmi. "Yo me bailaba como una mujer igualito... y vino a la casa de danzas un fiscal

10 Otras representaciones de vírgenes aparecen con la cara tapada, como en Cocharcas, Apurímac. joven... se me ha enamorado pe ... quería bailar no más pe... y yo como bailaba como una mujer también igualito, se me ha enamorado pe. Me hablaba pe... 'que sabes qué, que esto, que aquello, pucha. Quiero tener pareja contigo', 'ya ya' disimulaba... entonces al caporal le había dicho 'ya pe ¿cuántas cajas de cerveza?' El caporal le había dicho 'llévatelo a la imilla, pero pon 10 cajas'... empezaron a tomar, mientras estaban tomando saqué la tela negra, ha chocado con varón pe» (Testimonio de Francisco). De otra imilla se cuenta que «un diputado... se enamoró pues profundamente con esa imilla... el momento de comerse la merienda lo decimos 'si tú quieres, anda pon los, o sea ya pue, hacer sexo; anda con la imilla, autorizado anda.' Entraron al baño y la imilla levanta su falda, empieza a orinar. Hizo una chapada... era Andrés Quito, un señor imilla que hasta ahora nadie le iguala» (Testimonio de Braulio). El aspecto de $k a$ rihuarmi de la imilla está también presente cuando mujeres encarnaban al personaje, adaptándose a la compañía varonil. «Ellos en esta danza todos toman, entonces a mí me gustaba también, tomaba con ellos, 
hacía bromas... me admiraban porque a mí me decían. 'Oye, ¿es cierto? ¿esa es mujer o es varón?'... porque yo daba igual que el varón... mujer-hombre, el mismo patrón» (Testimonio de Margot).

Vemos que el aspecto del karihuarmi es esencial para el análisis de la imilla, también cuando la encarnan mujeres. Así que habrá que profundizar el tema de género con respecto a la imilla y su simbolismo.

\section{Andrógino, transgénero y travesti en los Andes}

Históricamente, otros personajes femeninos, como la china saqra, mujer demonio que acompaña a los Saqras, estaban encarnados por hombres. Aunque algunas mujeres representaron la imilla durante los años 90 del siglo pasado, varones volvieron a encarnarla. La explicación común refiere especialmente al carácter masculino de los Qollas, la resistencia corporal necesaria en el peregrinaje a Qoyllur Rit'i y en el yawar unu. El papel de la imilla en este ritual demanda ambas habilidades, como una madre solícita con resistencia corporal de un hombre. Por lo tanto, características de ambos géneros, o transgéneros están encarnados en la imilla.

Andrógino en la mitología griega significa posesión simultanea de ambos sexos, que es igual a no poseer ningún sexo; para perpetuar lo andrógino tiene que dividirse en dos, femenino y masculino (Brisson, 2002). Para los antiguos griegos, la unidad que se manifiesta por la sexualidad dual es perturbadora, expresa caos. La separación asegura el orden social, cosmológico y teológico (Brisson, 2002). En el Perú prehispánico, esta unión se expresó en esculturas andróginas (Classen, 1990), en el concepto bisexual del creador Wiracocha (Classen, 1990; Isbell, 1976) y en el wari, el ancestro del rebańo (Isbell, 1976). Este concepto, andrógino, existió ya en la cultura Chavín y se adaptó por los incas en su organización social y espacial como una forma de crear ideologías de jerarquía que expresaron las divisiones de la comunidad (Silverblatt, 1987: 40-66), así los hombres de hanan Cusco se consideraban masculinolmasculino, y los de hurin, masculinolfemenino, mientras las mujeres en hanan eran femeninolmasculino y en hurin eran femeninolfemenino (Rostworowski, 1989, citado por Campuzano, 2009: 83). En el contemporáneo Amarete, Bolivia, el género simbólico es adjuntado según los papeles sociales (Rosing, 1997). En los mitos de Huarochirí cuando un miembro de la élite falleció, se transformó en dos: mallqui y huaca ${ }^{11}$. La dualidad mallquilhuaca es un andrógino de la vida post mortem de la persona (Isbell, 1997). Así lo andrógino en los Andes es fundamental para la continuación de la vida y la organización social como un complemento que es indispensable (Isbell, 1976).

Actualmente lo andrógino en el Perú parece connotar una inversión de papeles que está relacionado mayormente a la reproducción agrícola. Durante la herranza de agosto en Huancavelica, una pandilla es guiada por un personaje travesti llamado Marín (Hernández Lefranc, citado por Campuzano, 2009). En los años 70 en Ayacucho, durante las ceremonias de yupay de agosto, después de la fecundación de la tierra, los hombres personificaban a las mujeres simbólicamente. Los warmis masculinos pintaban su cara de negro, parodiaban la fuerza de reproducción de las mujeres (Isbell, 1997) ${ }^{12}$. Después de la sequía, en Puquio, hombres y mujeres intercambiaban ropa y danzaban (Arguedas, 1964). Festividades de inversión están presentadas también durante carnavales en febrero, donde se concluye con una batalla entre los sexos (Ackerman, n.d) y un personaje travesti. En comparación al tinkuy, donde la separación sexual es manifestada, aquí el caos se celebra con una lucha de juego entre los sexos que puede concluir con violencia (Isbell, 1997). Festividades de inversión se relacionan con la fertilidad de la tierra, como en febrero, el mes más fértil (Ackerman, n.d), mientras que agosto se considera el más peligroso (Isbell, 1997) ${ }^{13}$.

La historia colonial del género en los Andes constituye una batalla entre las ideologías cristiana y andina, en donde las estructuras tradicionales de género se alteraron mientras los españoles discriminaron las posiciones políticas y religiosas de la anteriormente autoridad dual (Silverblatt, 1987). Isbell (1997) pregunta cuál es el destino del deseo sexual femenino cuando se encuentra con la ideología de virginidad.

11 Mallqui es la momia y su mitad femenina, que representa la semilla de las futuras generaciones. Huaca, la mitad masculina, es una roca que representa la fuerza inseminadora.

12 Un aspecto similar que parece estar relacionado al simbolismo de inversión aparece durante la limpia de acequias en Yauyos, Lima, y vinculado a otro simbolismo de inversión, los negritos (Hernández Lefranc, citado por Campuzano, 2009).

13 En el Cusco otro personaje travesti, la waylaka, acompańa una tropa de Qollas en Qoyllur Rit'i, además performa individualmente haciendo bromas y bailando con el público. 
La pregunta debe ser examinada por la comparación de la warmi con la imilla. Aunque en ambos casos un personaje andrógino está involucrado y representa un simbolismo de reproducción femenina, presentan papeles opuestos. La warmi y sus parecidos representan el caos de la tierra y de la reproducción social, mientras que la imilla representa un papel mediador entre mitades opuestas, significando otros aspectos de reproducción femenina, influenciada por la connotación de la Virgen; junto al andrógino, como símbolo de transición hacia lo sagrado (Ackerman, 1991), juntando así símbolos sagrados católicos y andinos. La Virgen en el catolicismo es considerada como mediadora entre los humanos y Dios. La imilla realiza el papel mediador con las representaciones de sus varias interpretaciones, y media entre los Qollas en el yawar unu, separando a los hermanos, y también en su posición entre las líneas de los Qollas. En la guerrilla, la imilla media entre los Qollas y los Ch'unchus. En esta representación la imilla no solamente encarna a la Virgen del Carmen, sino encarna la totalidad del concepto de Paucartambo, como mediador. Como he discutido anteriormente, es la construcción social y espacial de los Incas de hanan-hurin (Zuidema, 1964), con el templo del sol en el centro, reflejando así la división cosmológica, igual a la estructura del cuerpo con sus órganos vitales al medio (Classen, 1990). Así, usando los términos de Girard (2005), el tinkuy histórico de Qollasuyo\Antisuyo encontró su solución de violencia sagrada encarnada en la fiesta de la Virgen del Carmen, creando un campo de armonía social, reemplazando así la violencia contagio$\mathrm{sa}^{14}$. Ahora bien, argumento que la imilla es raptada en la guerrilla por el hecho que ella, con su extrema liminalidad, realiza un papel mediador en los diferentes niveles simbólicos de la fiesta de la Virgen del Carmen. Su rapto por los Ch'unchus representa la armonía social que el ritual en su totalidad pretende mantener.

La liminalidad de la imilla proviene del proceso ritual y del travestismo del personaje, y así juega un papel simbólico esencial en la fiesta de Paucartambo.

Travestis y transgéneros, entre otros personajes liminales, como ladrones y trabajadores de la industria de sexo, forman los devotos del culto de Sarita

14 Un caso similar sucedió en el santuario del Seňor de Justo Juez (Sallnow, 1987: 136-140).
Colonia, Lima. Campuzano (2009) entiende a Sarita Colonia como resistencia cultural a la herencia del sistema colonial que no permitió la continuidad de las representaciones travestis en los Andes. El culto también ofrece a sus devotos marginales una alternativa a las instituciones religiosas, además de la creación de un intercambio socioeconómico alternativo (Roper, 2014). Distinto de Cánepa (1998), que entiende la fiesta de Paucartambo como una representación de la identidad mestiza del pueblo, Vásquez ${ }^{15}$ argumenta que es una manifestación de resistencia a la opresión colonial por una aparente adaptación de costumbres foráneas mientras presentan costumbres prehispánicas. La imilla, como representante de las mujeres del Qollao, apoya otro símbolo de resistencia, así como las mujeres del Perú colonial huían a la puna, rechazando la opresión de la nueva ideología (Silverblatt, 1987). Además, Isbell (1976) argumenta que en los Andes la mujer es percibida como un elemento más poderoso en el proceso procreativo, $y$ que el modelo cosmológico complementario funciona como un equilibrio entre los sexos. Por lo tanto, la imilla como un personaje transgénero puede ser comprendida como una performance de resistencia cultural; primeramente, al proceso colonial que mutó al concepto de división espacial y social prehispánico, y por otro lado al proceso contemporáneo que demanda cambios en los papeles de género en la sociedad paucartambina y en su fiesta.

La imilla de Paucartambo fue tradicionalmente encarnada por danzantes varones hasta los años 90 . La decisión de aceptar mujeres no fue simple; pero el alcalde de esos años cuenta que los hombres "no querían bailar, o sea tenían una vergüenza» (Testimonio de Braulio). Esa situación lo obligó a buscar mujeres que presenten la imilla. Según los Qollas, la danza demanda habilidades masculinas y exige resistencia física, lo cual no es apto para una mujer. Según el alcalde, bajo su autoridad las mujeres presentaron "todo igualito como la tradición paucartambina lo instituía, ¿no? Ser una persona cabal, consciente de sus actos, lo hace todo que no tenga antecedentes penales. Así es la condición de un paucartambino» (Testimonio de Braulio). Esta declaración debe ser entendida en dos niveles: primero, el danzante tiene

15 Con su alma india, pero (Paucartambo, Cusco 1992). https://www. youtube. $\mathrm{com} /$ watch? $\mathrm{v}=\mathrm{dA} 2 \mathrm{rdEk} 8 \mathrm{Pxs} \& \mathrm{t}=113 \mathrm{~s}$ 
que realizar el personaje esperado. Segundo, sus actos como alcalde están tomados bajo una autoridad legal y así lleva responsabilidades. Ambos significados están señalados contra las críticas de los Qollas sobre el acto de aceptar mujeres como imilla. El anterior alcalde aceptó a una mujer por unas horas, cuando la imilla no podía participar: "una vez me había traído a una mujer vestida de imilla... y la agarré pe y era mujer, la metí adentro y le dije ' ‘a ti quién te ha dado permiso?' y me dijo 'no, la imilla ha venido a suplicarme'... y como que ya estaba vestido, le acepté hasta las 12 no más... y al año siguiente quería, no lo acepté... da pena pue, como a una mujer le voy estar dándole (en yawar unu), porque el Qolla es Qolla y tiene que darle duro, porque si no suena el caporal tiene que aumentarle... desde esa vez (que aceptaron) ya malogró la danza pe. Claro porque la imilla siempre ha sido antes también un varón, no mujer» (Testimonio de Amador).

Las mujeres que participaron de imillas son Luz Marina Multof, Ana Vargas, Olga Palacios, Margot Álvarez y María del Carmen Álvarez, bajo la autoridad de tres alcaldes. La participación de las mujeres en Paucartambo no duró mucho y el intento de cambiar la presentación tradicional no se extendió. Aunque los informantes argumentan que la presentación no se modificó por el cambio del género del danzante, y que las mujeres han participado igualmente en el yawar unu, este no es el caso en otras tropas ${ }^{16}$.

Aunque Cánepa (1998) argumenta que esta situación de género refleja una sociedad tradicional, donde las mujeres son discriminadas, lo opuesto sucede en Paucartambo. Varios karguyok en la fiesta son mujeres, como la karguyok de 2017 de los Qollas. El karguyok en la fiesta requiere de una inversión económica significativa: la renta de la casa de cargo, el pago a los músicos y cocineros, y la compra de la comida. La karguyok está siempre presente durante la fiesta, y aparece cerca al alcalde y la imilla durante la presentación y en el kargohuasí. Además, parece que las paucartambinas ocupan un papel importante dentro del pueblo; muchas administran el negocio mientras que sus esposos están menos activos corporal y

16 En Qoyllur Riti ivarias tropas de Qollas incluyen mujeres como imillas. Aparte de la falta de la tela negra en algunas, a veces más de una imilla participa. En esas tropas la imilla azota al alcalde en el yawar unu, pero él evita de azotarla. Además, cuando la imilla tiene que intervenir, un $u k u k u$ le reemplaza y separa los Qollas. verbalmente. Además, durante el último ritual de la fiesta, 19 de julio, el cambio de ropa de la Virgen está performado mayormente por mujeres. En las versiones de los Qollas que narran la llegada de la Virgen a Paucartambo, se involucra la influencia de paucartambinas pudientes (Villasante Ortiz, 1989); eso puede explicar la presencia de la imilla en la tropa y el papel importante de las mujeres en el sistema social y religioso del pueblo. Así, los Qollas sirven como un espacio de participación y resistencia a este proceso, agregando características masculinas que están mantenidas con requisitos exclusivos.

\section{Conclusión}

El personaje de la imilla puede ser interpretado de una manera distinta de la propuesta en esta publicación, por un discurso contemporáneo de género, travestismo y transgénero (Butler, 1999), especialmente en el Perú (Campuzano, 2006; 2009). Aquí he discutido sobre el personaje de la imilla como una performance de genero constitutivo de la fiesta de la Virgen del Carmen de Paucartambo, Cusco, y que tiene entre otros, un carácter político. Y en este sentido lo travesti, para esta interpretación, no está relacionado con la identidad de género y mucho menos con la orientación sexual. La imilla constituye una performance cultural que forma un caso único, como un ritual desarrollado en un ambiente particular. Por eso merece ser estudiado y explicado más que todo como ritual por derecho propio (Handelman, 2004). Así, la imilla y los Qhapaq Qolla son la representación fiel de su entorno, reflejándolo y modelándolo.

El fenómeno de la imilla es único en la fiesta de la Virgen del Carmen de Paucartambo. Es un personaje complejo, que refleja en muchos aspectos el carácter liminal de los Qhapaq Qolla. La imilla tiene varias interpretaciones, las que varían según la época, el danzante que la encarna o la observa. Uno de sus simbolismos es la encarnación de la Virgen del Carmen. Argumento que como un personaje transgénero la imilla no solamente encarna a la Virgen, sino también encarna la totalidad del concepto de Paucartambo como un núcleo, así como está narrado por los mitos y performado durante la guerrilla como encuentro de opuestos. Paucartambo \la Virgen del Carmen \la imilla median entre fuerzas duales en el 
contexto local. Así, la imilla se distingue y constituye un caso único entre los personajes travestis y transgéneros que representa una interpretación del tema de género en los Andes.

\section{Bibliografía}

Ackerman, R. (1991). The despacho: analysis of a ritual object. Journal of Latin American Lore 17: 71-102.

Ackerman, R. (n.d). Parody in Andean ritual (1-14). Unpublished manuscript. Los Angeles, CA: California State University.

Arguedas, J. M. (1964). Puquio, una cultura en proceso de cambio. In L. E. Valcarcel (ed.) Estudios sobre la cultura actual del Peru (221-272). Lima: Universidad Nacional Mayor de San Marcos.

Bataillón, M. (1995). Por un inventario de las fiestas de moros y cristianos: otro toque de atencion. In M. Bataillon \& A. Tauro del Pino (Eds.) La colonia, ensayos peruanistas (193-202). Lima: Universidad Nacional Mayor de San Marcos.

Brisson, L. (2002). Sexual ambivalence: Androgyny and hermaphroditism in Graeco-Roman antiquity. Berkeley, CA: University of California Press.

Butler, J. (1999). Gender Trouble: Feminism and the Subversion of Identity. New York-London: Routledge.

Campuzano, G. (2006). Reclaiming Travesti Histories. IDS Bulletin 37(5): 34-39.

Campuzano, G. (2009). Andróginos, hombres vestidos de mujer, maricones... el Museo Travesti del Perú. Baguas 4: 79-93.

CÁnePA, G. E. (1998). Máscara, Transformación e Identidad en los Andes: la fiesta de la Virgen del Carmen en Paucartambo-Cuzco (1a Edición). Fondo Editorial de la PUCP.

Classen, C, V. (1990). Inca cosmology and the human Body. Montreal, Canada: McGill University.

Classen. C., \& Howes, D. (2014). Ways of sensing: understanding the senses in society. New York: Routledge.

Coleman, S., \& Eade, J. (2004). Reframing pilgrimage: Cultures in motion. London; New York: Routledge.

Duviols, P. (1977). La destruccion de las religiones andinas: conquista y colonia. Mexico: Universidad nacional autonoma de Mexico.

Figueroa, L., Nishiyama, E., \& Villanueva, C. (Directors). (1961). Kukuli [Motion Picture]. Peru: Cine Club Cusco.
Girard, R. (2005). Violence and the sacred. New York. N.Y.: Continuum.

Gow, R., \& Condori, B. (1982). Kay Pacha. Cuzco: Centro de Estudios Rurales Andinos «Bartolome de las Casas».

Handelman, D. (1977). Play and ritual: complementary frames of metacommunication. In A.J, Chapman \& H. Foot (Eds.) It's a funny thing humor (185-192). London: Pergamon.

Handelman, D. (2004). Introduction: Ritual in its own right. Social Analysis 48(2): 1-32.

Hastrup, K. (1998). Theatre as a site of passage: some reflections on the magic of acting. In F. Hughes-Freeland (ed.) Ritual, performance, media (29-45). London: Routledge.

Hopkins, D. (1982). Juego de enemigos. Allpanchis Phuturinqa 20: 167-187.

Isbell, B. J. (1976). La otra mitad escencial: un studio de complementariedad sexual andina. Estudios Andinos 5(1): 37-56.

IsBeLl, B. J. (1997). De inmaduro a duro: lo simbólico femenino y los esquemas andinos de género. In D. Y. Arnold (ed.) Más allá del silencio: las fronteras de género en los Andes (253-301). La Paz: Biblioteca Andina.

Itier, C. (2004). Karu ñankunapi. Cuzco: CBC.

LÉvi-Strauss, C. (1966). The savage mind. Chicago: University of Chicago Press.

LÉVI-STRAuss, C. (1987). Introduction to the work of Marcel Mauss. London: Routledge \& Kegan Paul.

Millones, L. (1973). Un movimiento nativista del siglo XVI: el Taki Ongoy. In J. Ossio (ed.) Ideología mesiánica del mundo andino: antología (85-93). Lima: Ignacio Prado pastor.

Morote Best, E. (1988). Aldeas sumergidas: cultura popular y sociedad en los Andes. Cusco: Tall. Gráficos del Centro Bartolomé de Las Casas.

Muller, T., \& Muller, H. (1984). Mito de inkarri-qollari (cuatro narraciones). Allpanchis Phuturinqa 23: 125-143.

Murra, J. (1997). Existieron el tribute y los mercados en los Andes antes de la invasion europea? In R. Varon Gabai \& J. Flores Espinoza (Eds.) Arqueologia, antropologia e historia en los Andes: homenaje a Maria Rosworowski (737-748). Lima, Perú: Instituto de Estudios Peruanos: Banco Central de Reserva del Perú.

Pease, F. (1973). El Dios creador andino. Lima: Mosca Azul. Platt, T. (1987). The Andean soldiers of Christ. Confraternity organization. The mass of the sun and the regenerative warfare in rural Potosi (18th-20th 
centuries). Journal de la Societe des americanistes 73: 139-191.

Poole, D. (1982). Los santuarios religiosos en la economía regional andina (Cusco). Allpanchis Phuturinqa 19: 79-116.

Poole, D., \& Zuidema, T. (1982). Los límites de los cuatro Suyos incaicos en el Cuzco. Boletín del Instituto Francés de Estudios Andinos 11(1-2): 83-89.

Roper, D. M. (2014). Sarita Colonia y sus devotas travestis. Unpublished manuscript. New York, NY: New York University.

Rosing, I. (1997). Los diez generous de Amarete, Bolivia. In D. Y. Arnold (ed.) Más allá del silencio: las fronteras de género en los Andes (77-92). La Paz: Biblioteca Andina.

Sallnow, M. J. (1987). Pilgrims of the Andes: Regional cults in Cusco. Washington, D.C.: Smithsonian Institute Press.

Silverblatt, I. (1987). Moon, sun, and witches: Gender ideologies and class in Inca and colonial Peru. Princeton, N.J.: Princeton University Press.

Sklar, D. (2001). Dancing with the Virgin: body and faith in the fiesta of Tortugas, New Mexico. Berkeley, Los Angeles, London: University of California press.

Urton, G. (1993). Moieties and ceremonialism in the Andes: the ritual battles of the carnival season in southern Peru. Senri ethnological studies 37: 117-142.
Turner, V. (1982). From ritual to theatre: The human seriousness of play (Performance studies series; v. 1). New York: Performing Arts Journal Publications.

Van Den Berghe, P. L. \& Primov, G. P. (1977). Inequality in the Peruvian Andes, class and ethnicity in Cuzco. Columbia \& London: University of Missouri Press.

Gennep, A. van. (2004). The rites of passage. London, New York: Routledge.

VÁsquez, C. (2013a, June 25). "Con su alma india, pero» (Paucartambo, Cusco 1992) [Video file]. Retrieved from. https://www.youtube.com/ watch?time_continue $=113 \& \mathrm{v}=\mathrm{dA} 2 \mathrm{rdEk} 8 \mathrm{Pxs}$

Villasante Ortiz, S. (1989). Paucartambo provincia folklorica: Mamach Carmen, tomo II. Cusco: concytec (2nd edition).

Wachtel, N. (1973). La vision de los vencidos: la conquista espaňola en el folklore indigena. In J. Ossio (ed.) Ideología mesiánica del mundo andino: antología (3781). Lima: Ignacio Prado pastor.

Zuidema, R. (1964). The ceque system of Cuzco, the social organization of the capital of the Inca (International Archives of ethnography). Leiden: E.J. Brill.

Zuidema, R. (1991). Batallas rituales en el Cuzco colonial. In R. Thiercelin (ed.) Cultures et sociétés: Andes et MésoAmérique: mélanges en hommage a Pierre Duviols (811834). Aix-en-Provence: Université de Provence. 